\title{
Repensar la intervención social con grupos: premisas y orientaciones para una práctica transformadora
}

\author{
Alejandro Martínez González ${ }^{1}$
}

Recibido: 06/03/2017 / Revisado: 17/03/2017 / Aceptado: 06/07/2017

Resumen. Este artículo teórico propone una revisión de la intervención social con grupos, especialmente de los denominados socioeducativos y socioterapéuticos, con el objeto de ofrecer algunas orientaciones que ayuden a guiar y mejorar su práctica. Parte de la reconsideración de las aportaciones que más contribuyeron a construir el cuerpo teórico de la disciplina del trabajo con grupos, frecuentemente desconocidas por los profesionales de la intervención social, así como de la identificación de algunas de las acciones que más limitan sus posibilidades, entre las que se encuentra su simplificación. Y centra para ello especialmente su atención en la descripción de un conjunto de aspectos trascendentales para la comprensión de sus procesos y a los que, sin embargo, apenas aluden los materiales que pretender guiar el trabajo social y educativo con los mismos. El encuadre y la diferencia entre grupo y agrupamiento, el concepto de matriz grupal, la determinación de la tarea, el tránsito de la dependencia a la interdependencia, la consideración del poder y la pertenencia y la posibilidad de la complementariedad de roles, se presentan así como elementos ineludibles para un verdadero acompañamiento grupal transformador.

Palabras clave: Trabajo Social con grupos; intervención grupal; Educación Social con grupos; grupos socioeducativos; grupos socioterapeuticos.

\section{[en] Rethinking social intervention with groups: premises and guidelines for a transforming practice}

\begin{abstract}
This theoretical article proposes a review of social intervention with groups, especially the so-called socioeducational and socio-therapeutic, in order to offer certain guidelines that help guide and improve their practice. Part of the reconsideration of the contributions that contributed the most to the theoretical body of the discipline of working with groups, often unknown by social intervention professionals, as well as the identification of some of the actions that most limit their possibilities, its simplification included. And due to this, the focus is especially on the description of a set of transcendental aspects for the understanding of its processes and those that, however, are barely mentioned in the materials that seek to guide social and educational work with them. The framing and the difference between group and grouping, the concept of group matrix, the determination of the task, the transition from dependence to interdependence, the consideration of power and belonging and the possibility of the complementarity of roles, are presented as well as unavoidable elements for a true group support change trigger.
\end{abstract}

Key words: Social Work with group; Group intervention; Social Education with groups; Socio-educational groups; socio-therapeutic groups.

Sumario: 1. Introducción. 2. Algunos antecedentes y precedentes. 3. Aspectos esenciales para afrontar el trabajo con grupos. 3.1. El encuadre de grupo o agrupamiento. 3.2. El grupo como lugar seguro: la construcción de la matriz grupal. 3.3. La tarea grupal. 3.4. De la dependencia a la interdependencia. 3.5. El poder y la pertenencia. 3.6. Hacia la complementariedad de roles. 4. Conclusiones. 5. Referencias bibliográficas.

Cómo citar: Martínez González, A. (2018) Repensar la intervención social con grupos: premisas y orientaciones para una práctica transformadora, en Cuad. trab. soc. 31(2), 369-379.

\footnotetext{
Centro Superior de Estudios Universitarios La Salle España

alejandromg@lasallecampus.es
} 


\section{Introducción}

A menudo la intervención social y educativa en grupo se ha planteado como una oportunidad capaz de enriquecer y ampliar las posibilidades de las acciones o los acompañamientos terapéuticos y educativos individuales. Desde que en 1939 se creara la Asociación Americana para el estudio del Trabajo de Grupo (AAETG) y en 1946 el Trabajo Social con Grupos se considerase como un método específico del Trabajo Social (Rossell, 1998), son múltiples las justificaciones y aportaciones que subrayan sus posibilidades como vía o herramienta eficaz para la superación de las dificultades de las personas y la mejora de su vida en sociedad.

En este artículo se abordan algunos de los aspectos relacionados con dichas posibilidades a partir de la identificación de las contribuciones teóricas más significativas en torno al trabajo con grupos, deteniéndonos en aquellas que, desde nuestra experiencia en la intervención en grupos y la supervisión de equipos, se consideran de mayor relevancia y complejidad. Para ello, debemos empezar clarificando que cuando nos referimos a la intervención social con grupos estamos aludiendo a la posibilidad de una práctica en la que profesionales del Trabajo Social, la Educación Social, la Psicología, la Pedagogía, la Sociología y la Terapia Ocupacional entre otras disciplinas, procuran promover el logro de objetivos terapéuticos o educativos en un conjunto de personas que coinciden en un interés, una necesidad o un daño, para cuyo abordaje o superación son congregadas en una labor conjunta. Una labor asumida habitualmente en grupos pequeños, de entre cinco y 15 personas, pero que, como en los casos de los encuadres multifamiliares (García Badaracco, 2000), pueden llegar a ser incluso de varias decenas.

Centra, por tanto, su atención fundamentalmente en los grupos denominados socioeducativos y socioterapéuticos, entendiendo los primeros como los que se convocan para atender una necesidad formativa y los segundos como los constituidos para compartir y abordar un padecimiento o una herida singular, pero con rasgos comunes (Rossell, 1998, 2014).

\section{Algunos antecedentes y referentes}

Tres serían los campos en los se sitúan los tipos de intervenciones grupales a los que nos referimos: el educativo, el social y el psicoterapéutico. Con respecto a sus precedentes, podemos decir que el del educativo lo establecen las organizaciones juveniles de educación no formal como YMCA o el movimiento Scout, surgidas a mediados del siglo XIX y comienzos del XX respectivamente, para promover el desarrollo integral de los y las jóvenes a través de prácticas colaborativas en el medio natural durante su tiempo libre y de ocio.

El del campo de la ayuda social, se sitúa en los Settlement Houses (Tannenbaum \& Reisch, 2001), los centros sociales comunitarios que se implantan en Inglaterra y Estados Unidos a finales del siglo XIX, cuya labor se orientaba a buscar soluciones para las familias sin recursos, frecuentemente inmigrantes, a través del trabajo en grupo o de la organización comunitaria, y que instituirán una nueva forma de hacer que considera el encuentro y el apoyo mutuo como oportunidades para la integración social.

Con respecto al campo psicoterapéutico, será el trabajo de Josph H. Pratt a partir de 1905, con sus clases colectivas de pacientes tuberculosos en el Massachusetts General Hospital (Sánchez-Escárcega, 2006; Vives Belmonte, 2014), el que marcará el punto de partida del uso del grupo como recurso para abordar y procurar sanar o paliar el padecimiento psíquico, utilizando las emociones colectivas con una finalidad terapéutica.

Junto a ellos, merece también una atención específica J. L. Moreno, que en 1921 inicia la práctica del psicodrama, una disciplina terapéutica que el propio autor considera "un punto de vista decisivo en el apartamiento del tratamiento del individuo aislado, hacia el tratamiento del individuo en grupos" (Moreno, 1993, p. 32) y cuyas propuestas y técnicas se recogen en buena parte del trabajo con grupos que se lleva a cabo en la actualidad, tanto en el ámbito terapéutico como en el educativo y en el social.

El fundamento teórico de la práctica grupal irá armándose a partir, por tanto, del primer tercio del siglo XX, amparado por los postulados de Rousseau y su consideración de la necesidad del otro para su complétude (Todorov,1995), de los de Durkheim y su concepción del grupo como totalidad, como algo más que la suma de sus miembros (Anzieu, 2007), de los de Dewey y su apuesta por la democracia como forma de relación interpersonal $\mathrm{y}$, muy especialmente, del interaccionismo simbólico 
de Mead y su defensa del origen social de la persona (Zamanillo, 2008).

De este modo, en la medida en que avanza la apreciación de la dimensión social del individuo, crecen también las prácticas profesionales con colectivos y grupos, así como la investigación sobre sus posibilidades y limitaciones y las aportaciones teóricas sobre los modos en que pueden llevarse a cabo.

Kurt Lewin se convertirá entonces en un referente inevitable (Burnes, 2004) al intentar en 1946, desde el Centro de Investigación para la Dinámica de Grupos del Massachusets Institute Technology (MIT), "la primera representación científica de los fenómenos grupales" (Sánchez-Escárcega, 2006), dando pie al concepto de dinámica de grupos y estableciendo como premisa para su comprensión el análisis de las interacciones.

A sus aportaciones se sumarán pronto las de W. R. Bion, que ya en 1948 desde la clínica Tavistock de Londres, comienza a trabajar con grupos de pacientes compuestos por militares con trastornos psíquicos, lo que le permitirá en los años 1960 dar forma a una teoría de fundamento psicoanálitico (Bion, 2004) con la que pretendía dar cuenta de lo que acontece al grupo como ente particular.

También Pichón Rivière, médico psiquiatra argentino, tras su labor en el hospital psiquiátrico de Las Mercedes en Buenos Aires entre los años 30 y 40, elaborará un importante constructo teórico sobre el acontecer de los grupos a partir de los años 1950, que girará en torno al concepto de grupo operativo y en el que convergen las influencias del psicoanálisis kleiniano con las de Mead, Lewin o Schilder (Fabris, 2007). Una apuesta de integración teórica que le conducirá a la expulsión de la Asociación Psicoanalítica Argentina que él mismo contribuyó a crear, al exceder sus límites doctrinarios. Algo similar a lo que le aconteció a otro referente más de la teoría de grupo, S. H. Foulkes, expulsado de la institución psicoanalítica cuando, en su ejercicio de la medicina, optó por ver pacientes conjuntamente con sus familias y en grupo. Una salida que le permitió crear su propio modelo de psicoterapias grupo-analíticas, que puso en práctica en el Hospital inglés de Northfield durante la Segunda Guerra Mundial con personas que padecían neurosis de guerra (Campos, 1986).

El campo teórico del análisis de los grupos se verá ampliado a partir de entonces tanto por las aportaciones de nuevos autores de orien- tación psicoanalítica como D. Anzieu, J. B. Pontalis, R. Kaës, A. Missenard o A. Bejarano (Sánchez-Escárcega, 2006); como por otros de perfil más humanista, como J.P. Sartre, C. Rogers, A. Maslow o el fundador de la escuela de terapia gestáltica F. Perls (López-Yarto, 2004). Autores de amplia relevancia a los que podemos sumar a G. Konopka (1968) por su esfuerzo pionero en la sistematización inicial del Trabajo Social con grupos a finales de la década de 1960 y, desde luego, a García Badaracco (2000) por el desarrollo y sistematización del trabajo socio-terapéutico con los denominados grupos multifamiliares en el Hospital Neuropsiquiátrico Borda, de Buenos Aires, desde 1960.

Este trabajo se fundamenta en las contribuciones de todos ellos, así como en los matices introducidos posteriormente por profesionales e investigadores como resultado de su reflexión en torno a la práctica tanto terapéutica como social o educativa en grupo.

Resulta paradójico sin embargo que, mientras en los contextos exclusivamente terapéuticos el cuerpo teórico que se ha constituido en torno a esta disciplina viene siendo considerado desde hace años, es aun frecuentemente desconocido o desconsiderado por buena parte de los y las profesionales de la acción social y educativa (López-Yarto, 2004; Garrett, 2005; Zamanillo, 2008; La Rocque, 2016). Unos profesionales cuya formación sobre el grupo y sus posibilidades parece haberse ido relegando (Carey, 2016) y cuya práctica en este ámbito queda, en muchas ocasiones, reducida a la implementación de paquetes de sesiones orientadas por manuales (Bergart, 2015) y centradas básicamente en promover la adquisición de pautas de conducta desde paradigmas individualistas (Parra, 2014). Algo que contribuye a su justificación bajo una premisa exclusivamente economicista: resulta rentable y menos costoso hacer con varias personas a la vez lo que con más recursos se haría individualmente, se viene a afirmar (Duro, 2002; Carey, 2016).

Y así, aun pretendiendo lo contrario, la intervención social con grupos corre el riesgo de terminar constituyendo una vía más de influencia y adoctrinamiento, un espacio al que las personas son convocadas, como dirá Anzieu (2007), más para observar la soberanía que para ejercerla. Algo que, entendemos, merma profundamente las constatadas posibilidades de transformación de la acción social con 
grupos (Drumm, 2006; Vinogradov \& Yalom, 2012; Parra, 2014).

\section{Aspectos esenciales para afrontar el trabajo con grupos}

Por este motivo, y con el ánimo de contribuir al reto de mejorar la eficacia de su práctica, pretendemos a continuación describir y destacar, desde un análisis e interpretación personal fundamentado en la propia praxis, aquellos aspectos que consideramos especialmente significativos para comprender y abordar los fenómenos más complejos que se producen en el seno de los grupos. Aspectos a los que, paradójicamente, apenas se presta atención en la mayor parte de los materiales que pretenden guiar la práctica de los y las profesionales. Unos materiales centrados, las más de las veces, en la mera recopilación de técnicas (Campos Vidal, 2000) o en la descripción taxonómica e inconexa de elementos que, siendo relevantes, como los objetivos y normas, las fases grupales, los roles, el liderazgo o el conflicto, son presentados frecuentemente de un modo tan simplificado que resulta imposible vislumbrar su utilidad para la intervención.

\subsection{El encuadre de grupo o de agrupamiento}

Como se puede deducir de lo anteriormente mencionado, entre convocar a las personas para aprovechar las ventajas de atenderlas agrupadas, y promover un proceso grupal hay un importante matiz diferencial. La clarificación de este aspecto es fundamental a la hora de abordar el trabajo social con grupos, por lo que la posibilidad de repensar su implementación encuentra en el encuadre de grupo o agrupamiento un importante punto de partida.

$\mathrm{Si}$ entendemos que trabajar con un grupo supone promover una acción cuya consecución entraña una necesaria interdependencia entre todas y cada una de las personas que lo integran (Papell, 2015), bien sea para la adquisición de un aprendizaje, bien para la atención de un padecimiento, todas las acciones que no cumplan este requisito se alejan de lo que dicen ser si se denominan a sí mismas intervenciones grupales. Y se alejan fundamentalmente porque consideran grupo a aquello que no cumple el requisito que debería ser indispensable para serlo: el nudo y lo redondo, como subrayará Anzieu (2007) acudiendo a la etimología de la palabra o, lo que es lo mismo, la cohesión, la reunión y la cooperación. El grupo al que nos referimos, que Bion conceptualizará como "grupo de trabajo" (2004, p. 116), no sólo precisa de objetivos comunes, sino de relaciones cooperadoras como vía para su consecución, y todo lo que no sea capaz de llegar a ese nivel queda reducido al campo del agrupamiento.

Desde esta idea, se considera trabajar desde el agrupamiento a cualquier acción en la que un profesional, bajo la consideración de su rol de experto, convoca periódicamente a un conjunto de personas únicamente para transmitirles un conocimiento, para indicarles un camino, para moldearlas o incluso para entretenerlas. Un modo de hacer que suele situar a los y las participantes en una permanente dependencia del o la dinamizadora, y que suele acarrear consecuencias como: una escasa participación, falta de compromiso, un constante desconocimiento mutuo de los y las participantes, mucha inhibición, un frecuente cuestionamiento soterrado de lo que se hace o se dice y la configuración de subgrupos por afinidades capaces de permanecer totalmente al margen unos de otros durante la actividad para la que son congregados. Situaciones todas ellas que, si bien se pueden dar también en un encuadre de grupo, como de hecho se dan, en éste se convierten en material de trabajo, en aspectos sobre los que pensar, mientras que en un encuadre de agrupamiento, en el que la consideración del grupo y su participación es meramente coyuntural, se perciben como desconcertantes y constituyen, como mucho, una ocasión para el reproche: "¿Es que nadie tiene nada que decir?", "Parece que no os interese lo que estamos viendo", "Hacemos esto por vosotros y no lo valoráis...", etc.

El trabajo desde un encuadre real de grupo requiere, por tanto, que los y las profesionales puedan comprometerse con todas y cada una de las personas que lo integran y contribuir a que se comprometan entre sí, siendo capaces de construir con ellas y ayudar a que construyan juntas (Roy \& Pullen-Sansfaçon, 2016).

Esta forma de entender la intervención grupal entraña una labor que requiere de las y los profesionales un profundo trabajo personal, pues no es fácil considerar en su particularidad a las personas con las que se trabaja en grupo y al tiempo verse separado o separada de ellas (Walling, 2012). Una separación que constituye un requisito indispensable para poder pedirles y darles, para poder decirles que sí y que no 
y para llegar a contribuir a que ellas mismas lo puedan hacer entre sí. Todo ello sin olvidar que cada componente del grupo es indispensable e inevitable.

\subsection{El grupo como lugar seguro: La cons- trucción de la matriz grupal}

Cuando la pretensión es la de abordar una tarea grupal como la que venimos describiendo, una parte importante del camino es la construcción de una matriz grupal, entendida ésta como un tipo de matriz social, como un "espacio de crecimiento en el cual el sujeto va adquiriendo nuevos roles" (López-Barberá \& Población, s.f., p. 11) gracias a su condición de útero o lugar protegido para el grupo. Un requisito trascendental que favorecerá que las personas que asisten puedan percibir que se encuentran en un contexto suficientemente seguro como para poder creer que es posible que se cumpla lo que esperan de él.

¿Será este el sitio que logrará contener mi angustia? ¿Realmente aprenderé aquí lo que quiero? ¿Tendré en este lugar la oportunidad de ser yo misma? Que la respuesta a estas cuestiones sea positiva dependerá, en buena parte, de la viabilidad de la tarea grupal. Por ello, tanto la organización como las figuras profesionales que la promueven deberán velar por hacerlo factible, a sabiendas de que, en ocasiones, los y las mismas participantes trabajarán, siquiera inconscientemente, para boicotearlo, como una forma de ponerlo a prueba. La organización será, en este sentido, la primera que se someterá al escrutinio de los y las integrantes del grupo en ese proceso de construcción de la matriz: ¿Se ha logrado otras veces entre estas paredes lo que yo vengo a buscar? ¿Son sus muros suficientemente resistentes?

Del mismo modo, el equipo profesional encargado de la dinamización del grupo será el siguiente en ser examinado: ¿Serán estas las personas que yo necesito? ¿Sabrán lo que dicen saber? ¿Han podido ayudar o enseñar realmente a otras personas como yo?

Institución y profesionales estarán inevitablemente bajo sospecha. Las personas que configuran el grupo mostrarán sus dudas y previsiblemente se situarán en una retadora posición de cuestionamiento y retirada. Una posición que, en cualquier caso, puede ser el resultado tanto de una buena labor profesional como de una poco acertada. De una buena labor porque una buena práctica ha de ayudar al individuo a poder quedarse sólo y sostenerse sin buscar la complicidad del grupo a cualquier coste. Y de una poco acertada si lo que se hizo fue promover la dependencia y negar la posibilidad de diferenciación. Saber si las resistencias surgen por una u otra razón será el fruto de la necesaria labor auto-reflexiva $y$ de supervisión (Carey, 2016) que entraña también el trabajo profesional con el grupo, del que dependerá que el proceso avance o se estanque.

El requisito para la existencia de una buena matriz, de un lugar que se perciba como seguro, donde el proceso grupal pueda iniciarse y desarrollarse, será precisamente la construcción de un espacio en el que pueda pasar, sin que se derrumbe, aquello que precisamente más se suele eludir: la discusión, el desencuentro, la crisis y el conflicto, en suma, la controvertida e inestimable diferenciación de los sujetos. Un espacio donde esté permitido disentir, donde poder estar solo, pero con los otros, porque estar con los otros no requiera sumisión ni exclusión, como suele ocurrir en los encuadres de agrupamiento.

\subsection{La tarea grupal}

La descripción de la tarea es un aspecto que habitualmente se aborda al comienzo de la configuración del grupo con una explicación por parte del equipo de profesionales sobre el fin y los objetivos de su convocatoria y que parece quedar así zanjado. Sin embargo, nada más lejos de la realidad, fundamentalmente porque abarca no sólo su descripción, sino el proceso que entraña su concreción: la tarea es también la propia determinación y revisión de la tarea. Una labor que atañe a todo el grupo y que se extiende a lo largo de todo el proceso grupal. Lo que implica inevitablemente el análisis en grupo de las relaciones que se dan en su propio seno.

Consideramos así que la tarea, cuando se trata de abordar el trabajo con grupos socioterapéuticos y socioeducativos, pasa por la superación del malentendido que suscita su propia convocatoria: el de presuponer que serán los y las profesionales quienes sanen o resuelvan las dificultades de las personas que demandan ayuda. Pues bien al contrario, su éxito radicará precisamente en que se engendre la posibilidad de que sea cada sujeto el que llegue a hacerse cargo de sí mismo, saliendo así de la dependencia de quien toma como referente. 
En la medida en que entendemos que la tarea alude tanto a la meta como al recorrido que habrá de transitar el grupo para alcanzarla, requerirá que, en algún momento, éste pueda enfrentarse a cuestiones como las siguientes: ¿Para qué estamos aquí?¿Queremos realmente todos y todas ir en la dirección que llevamos? ¿Lo estamos haciendo? Y si no es así ¿qué nos está impidiendo hacerlo?

Preguntas sencillas pero trascendentales que los y las profesionales tendrán que poder trasladar al grupo tantas veces como intuyan que éste se distrae de su cometido. Algo que le puede estar ocurriendo cuando divaga e intelectualiza en los debates, cuando posterga el comienzo de las sesiones, cuando se producen retrasos reiterados, cuando focaliza las críticas en una persona o cuando se normalizan las ausencias. Modos de hacer todos ellos que, hipotéticamente, pueden funcionar como mecanismos de defensa del grupo en su conjunto, o de alguno de sus miembros en particular, frente a las dificultades que lleva implícita la labor a la que nos venimos refiriendo de hacer con los otros.

En torno a esta cuestión, la de los mecanismos de defensa del grupo frente a la tarea, Walter Bion (2004) construyó una trascendental teoría que da cuenta de su pertinencia. Hacer con los otros es andar un camino por el que se avanza en la medida en que se pueden ir afrontando las resistencias inconscientes que, paradójicamente, el propio grupo irá generando para recorrerlo. Resistencias que él situará en tres formas de hacer complementarias, que denominará supuestos básicos, y que actuarán como barreras a la labor que el grupo debería asumir. Estas resistencias serían: a) la dependencia del coordinador o la coordinadora, de quien se esperará que establezca lo que hay que hacer y evite y resuelva las angustias grupales; b) el ataque a esta figura o al propio grupo, o la huida del mismo como medio para defenderse y evitar dichas angustias; c) y lo que él describirá como emparejamiento: la esperanza inconsciente de que el grupo sea salvado por la aparición de un Mesías, engendrado por él mismo, y capaz de resolver lo que le atrapa, de traer lo que le falta, de hacer lo que la figura de coordinación no está siendo capaz de hacer.

Algunos ejemplos de estas manifestaciones pueden ser:

- Las demandas reiteradas a las y los profesionales para que resuelva las disputas o los desencuentros: "Di tú quién tiene la razón", "decídelo tú que eres la que sabes."

- Los reproches que se le hacen por permitir que se haya producido la controversia: "Tú sabías que esto iba a pasar y no lo has evitado."

- Los retrasos redundantes e injustificados de miembros del grupo o su falta de asistencia.

- O, también, las manifestaciones que subrayan que el grupo funciona mejor cuando, ocasionalmente, la figura coordinadora se ausenta: "Hoy que no has estado, y que fulanita y menganito se han hecho cargo de tu papel, hemos trabajado mucho mejor".

La tarea grupal tendría que poder centrarse, por tanto, precisamente en lograr que el grupo reflexione en torno a las posibilidades de estar actuando bajo un supuesto básico favoreciendo la reflexión conjunta y el pronunciamiento de cada sujeto.

Y mientras esto no se pueda producir, el grupo permanecerá en un estado infantil de demanda y aglutinamiento, "como un grupo de niños que espera ser tratado por turno y en forma individual" (Grinberg, Sor \& Tabak, 1991, p.26). Manteniéndose así en una fantasía de fusión que hará sentir como idílica una coexistencia sin aparentes controversias ni conflictos, donde cualquier objeción será percibida como una amenaza. Lo que llevará a la descalificación de la persona que la enuncia: "Si no hubieras dicho nada, seguiríamos tan bien como estábamos"; en vez de considerarla como posible portavoz de un emergente grupal (Berstein, 1986), capaz de traer a colación lo que nadie quiere ver.

Estas resistencias inconscientes al cambio que entraña la tarea, Pichón Rivère (1981) las englobará bajo el concepto de pretarea, y se referirá al grupo capaz de afrontarlas y llegar a emprender un proyecto propio como Grupo Operativo. Este autor describirá la tarea como la construcción de un ECRO grupal, un esquema conceptual referencial operativo, que consiste en una forma de hacer propia del grupo, particular y única, armada por él mismo a partir de la consideración e integración progresiva de las capacidades y aspectos identitarios de cada persona que lo compone.

En este complejo proceso de construcción del ECRO grupal, la figura de coordinación tiene como labor acompañar al grupo para que pueda llegar a saber de su necesidad, sin caer 
en la tentación de imponer ella misma su propia visión.

\subsection{De la dependencia a la interdependen- cia}

La dependencia constituye en todo este proceso uno de los grandes ejes vertebradores de la intervención grupal. Está en el inicio de la mayor parte de las iniciativas que la promueven y constituye, al tiempo, el mayor obstáculo para su desarrollo. Ya hemos visto que opera como mecanismo de defensa del grupo frente al abismo de su autonomía.

La dependencia es una posición que el grupo, como el sujeto, se resiste a abandonar ante la dificultad que entraña el crecimiento y la separación, y en la que a veces también quedan atrapados los y las profesionales que lo acompañan, seducidos por los cantos de sirenas de sus componentes. Unos cantos de sirena que sitúan a estas figuras en el lugar del padre o la madre, que subrayan su labor de creadores, de fuente nutricia insustituible, que les otorgan la primera y la última palabra.

De este modo, el grupo tiende a actuar inicialmente como un ente aglutinado que, poco a poco, y como fruto de un buen acompañamiento, deberá ir disgregándose, de manera que se puedan hacer visibles todos y cada uno de los sujetos que lo componen en su complejidad. Algo para lo que será fundamental la "acción liberadora del diálogo" (Freire, 2007, p. 181), por su capacidad para generar el tránsito de la obediencia a la emancipación.

Acompañar a un grupo en este sentido, supone permanecer atado al mástil para no verse arrastrado al mar de la seducción grupal, que se resiste a la diferenciación, y poder facilitar desde ahí que abandone el lugar de la demanda constante a las figuras de coordinación. Compartir pareceres, interrogarse, confrontar puntos de vista, lograr que afloren los desencuentros, son actos de autonomía que permitirán al grupo avanzar en ese trayecto para dejar de ser un todo indiferenciado, un mero agrupamiento, y poder pasar a ser interdependiente.

\subsection{El poder y la pertenencia}

El grupo, el tipo de grupo al que nos venimos refiriendo, es o habrá de ser, por tanto, un conjunto de fuerzas trenzadas capaz de actuar coordinadamente. Una capacidad, esta de la actuación coordinada, que precisa, como estamos viendo, de proceso, conciencia y entrenamiento.

Si nos detenemos ahora en lo concerniente a la conciencia, un aspecto fundamental en el que fijar la atención es el del poder ${ }^{2}$. El poder inherente de cada sujeto, como capacidad de acción individual y colectiva, pero también el poder como capacidad de alienación de los otros.

Con respecto al primero, al poder de emancipación de los sujetos, su fomento y desarrollo está en el sentido y fundamento de la mayor parte de las acciones sociales desde que el Trabajo Social lo incorpora a su razón de ser en los años 1960 con el movimiento reconceptualizador (Zamanillo \& Rodríguez, 1990).

Pero es en la dimensión del poder de dominación de los otros en la que más nos interesa detenernos ahora, ya que constituye su cara más invisibilizada. Como afirma Zamanillo: "El poder, al igual que la libertad, da miedo, confunde, se ve como algo que no se debe tener, que es algo propio de otros, que es malo" (2012, p. 160). Y así, se produce el fenómeno paradójico en la intervención social educativa y terapéutica de usarse y negarse al mismo tiempo, tanto en las relaciones entre iguales, como y, sobre todo, en la acción de los y las profesionales con las personas a las que acompañan.

Por eso, una parte importante de la dificultad del proceso que venimos describiendo radica precisamente en visibilizar su existencia para lograr que "el grupo desarrolle relaciones de poder" (Zamanillo, 2008, p. 138) capaces de autorregularse, con el objeto de que los sujetos no queden arrollados por las mismas.

Sobre las relaciones de poder que se producen entre los iguales en el seno de un grupo, podemos decir que alimentan su fragmentación, limitando así su evolución. Conducen

Un elemento trascendental en el análisis de las relaciones sociales abordado ya en la antigüedad por autores como Maquiavelo, Hobbes o Rousseau, y más recientemente por figuras referentes de la filosofía y la sociología como Weber, Nietzsche o Foucault (Zamanillo, 2012). 
habitualmente a la generación de subgrupos que se pueden armar por afinidad, pero también por oposición entre unos y otros, y en los que suele ser habitual la adscripción a ciertas figuras de liderazgo. Estas luchas de poder se arman precisamente en la competición de estas figuras y sus afines por capitanear los procesos o adaptarlos a sus propias necesidades (De Robertis \& Pascal, 1994), guiados por la consecución de sus intereses particulares. Intereses que, a veces, por lucha por el status o por incompatibilidad entre los miembros (Shaw, 1976), radican meramente en boicotear los de los otros (Cembranos \& Medina, 2003).

Por ejemplo, puede pasar que las personas más comprometidas con la tarea que convoca al grupo perciban a las que muestran menos entusiasmo o colaboración como un lastre, mientras que estas otras pueden sentirse prejuzgadas y rechazadas alejándose de las primeras y sus entusiasmos. Se activa de este modo una espiral en la que los discursos se realimentan y las partes se distancian progresivamente. Algo que nutre un modo de actuar irreflexivo y tentador para todos y todas, pues garantiza la presencia de un otro persecutorio en el que depositar la culpa, evitando tener que interpelarse sobre la propia necesidad de pertenencia y su coste (Pereña, 2010).

Con relación al uso del poder de los y las profesionales, nos interesa aquí subrayar la tendencia que les conduce a eludirlo rechazando su existencia (Idareta, 2017). Una posibilidad que se da cuando se aborda la labor profesional desde un bienintencionado igualitarismo, desde una horizontalidad mal entendida, que les lleva a afirmar que no existen diferencias porque todos y todas somos iguales.

Una sentencia que es tan cierta como falsa. Cierta porque no se puede negar que la condición de personas nos iguala a todos y todas, pero falsa porque desconsidera una evidencia: profesional y participante no son iguales porque en un encuadre clásico de intervención la regulación y control del mismo corresponde al primero y eso le otorga un poder que el segundo no tiene y del que ha de hacerse cargo por responsabilidad (Bertomeu, 2000; Pelegrí, 2004). Un poder al que además no debe renunciar "si no quiere privar al grupo de una de las más necesarias experiencias: la de la rebelión y la independencia" (López-Yarto, 2004, p. 159).

Lo que no descarta, en cualquier caso, la posibilidad de su ejercicio de un modo demo- crático y deliberativo, desde el que se contemple la cesión y reconocimiento de la parte posible del mismo al grupo en su conjunto y a cada uno de sus integrantes en particular, dando así cabida a su problematización, que constituye un requisito básico para la salida de la dependencia y la sumisión.

En este sentido, la horizontalidad, desde donde nosotros la entendemos, no pasa así tanto por la negación del poder de los y las profesionales, como por la prevalencia de la transparencia en su reconocimiento y por el compromiso de procurar entablar relaciones comunicativas donde prime el diálogo igualitario con voluntad de acuerdo, la acción comunicativa a la que se referirá Habermas (2010), en la que todas las opiniones tienen cabida y se argumentan sin recurrir a criterios de autoridad (Flecha, 1997), abriendo la puerta a una posible redefinición de las relaciones entre los y las profesionales y las personas destinatarias del recurso.

\subsection{Hacia la complementariedad de roles}

Moreno (1993) describirá el rol como "la forma de funcionamiento que asume un individuo en el momento específico en que reacciona ante una situación específica en la que están involucradas otras personas u otros objetos" (p. V). Una forma de funcionamiento donde se conjugan "la verticalidad del sujeto y la horizontalidad del grupo" sostendrá Berstein (1986, p.14), entendiendo la verticalidad como la historia de cada componente en la asunción de determinados papeles y la horizontalidad como lo que comparte el grupo.

Habitualmente la aproximación a la variable de los roles en los grupos se ha centrado en la mera identificación y descripción de la taxonomía de los diferentes modos de actuación, básicamente con el objeto de neutralizar a quienes representaban papeles considerados negativos para el grupo y reforzar a los que interpretaban los valorados como positivos. Sin embargo, esta es una mirada que entraña una profunda simplificación, básicamente porque parte del reduccionismo de considerar la parte por el todo. Cuando un sujeto se presenta ante los otros asume un modo de estar, una pose, y en la medida que ésta es confundida con la identidad misma, el individuo queda reducido a una etiqueta, a una particularidad que lo simplifica y descalifica. Este encasillamiento, al que Pichón Rivière se referirá como rol 
fijo, suele marcar y desgastar las interacciones en los grupos, y el reto fundamental de los mismos es trabajar para su superación: pasar de los roles estereotipados a otros funcionales, intercambiables y operativos (Berstein, 1986).

Así se refieren a este aspecto Herranz, Silva y Herranz (2014):

Si el cambio de roles llega a una posición ideal, la denominamos encuentro; entendemos que es la meta que, en algunas ocasiones, alcanzamos. Se parte de la posibilidad de una profunda comprensión del otro y se llega a un lugar no previsto de enriquecimiento mutuo que trasciende a las personas que forman parte de ese "momento". (p. 32)

El encuentro, el reconocimiento mutuo y la cooperación son, por tanto, el requisito, y el diálogo igualitario la vía para que se pueda producir. En su ejercicio y cuidado está la oportunidad de que el sujeto pueda pasar de estar a ser: abandonar la pose, su rol rígido, y compartir su particularidad, su rol operativo.

Un tránsito en el que la aceptación conduce a la complementariedad de roles (Zamanillo, 2008), donde los sujetos aprenden a servirse unos de otros y donde ser ellos mismos constituirá su principal oportunidad y contribución.

\section{Conclusiones}

Entendemos que la consideración de cada uno de estos aspectos contribuye a la mejora de la efectividad de las intervenciones socioeducativas y socioterapéuticas con grupos, pues permiten identificar elementos que resultan trascendentales para poder guiar su práctica, como son: la reflexión en torno al ejercicio del poder por parte de los profesionales de la intervención social frente a las personas a las que pretenden acompañar; el cuestionamiento de las acciones orientadas al adoctrinamiento; la consideración de los individuos en su particularidad; la construcción de la matriz grupal; la clarificación de la tarea; y la indispensable promoción del diálogo igualitario como vía para la acción.

Pasarlos por alto limita significativamente la eficacia de las intervenciones grupales, ya que reduce la posibilidad de tomar conciencia de su complejidad. Y cuando esto ocurre, cuando se simplifica la mirada, la acción grupal se reduce a la creación de espacios de obediencia, donde el camino marcado sólo deja sitio para el agrupamiento y la dependencia. Justo lo contrario de lo que se debería esperar de ella: que fuera capaz de promover una convocatoria donde, a través de la interacción, el diálogo y la cooperación, las personas se vean capaces de superar la sumisión y tengan la oportunidad de encontrarse con su propio deseo. Ésta habría de ser su principal contribución, su gran transformación, la de hacer de la reunión un lugar de paso al que pertenecer para emanciparse luego, pues es en el tránsito de este recorrido donde aparece una oportunidad para el cambio, que como sugiere Berstein (1986), es donde el aprendizaje y lo terapéutico se encuentran.

\section{Referencias bibliográficas}

Anzieu, D. (2007 [1997]). La dinámica de los grupos pequeños. Madrid, España: Biblioteca Nueva.

Bergart, A. M. (2015). Introduction to Part Three: The Relationship of Social Work with Groups to Group Work in Other Professions. Social Work with Groups, volumen 38, 253-254. Recuperado de: http:// www.tandfonline.com/doi/full/10.1080/01609513.2014.950969 Berstein, M. (1986). Contribuciones de Enrique Pichón Rivière a la psicoterapia de grupo. Clínica y Análisis Grupal, 39, 9-34.

Bion, W. R. (2004). Experiencias en grupos. Barcelona, España: Paidos.

Burnes, B. (2004). Kurt Lewin and the Planned Approach to Change: A Re-appraisal. Journal of Management Studies 41:6, 977-1001.

Campos, J. (1986). La orientación grupoanalítica en la formación de psicoterapeutas: el magisterio de S.H. Foulkes. En Martí Tusquets, J.L y Sadne, L. (Ed.) La formación en Psicoterapia de Grupo y Psicodra$m a$, 23-41. Barcelona: Argot.

Campos Vidal, J.F. (2000). Los factores terapéuticos: ¿Qué es lo que produce cambios en los grupos? Alternativas. Cuadernos de Trabajo Social, $\mathrm{n}^{\circ}$ 8, 205-229. 
Carey, L.A. (2016). Group Work Education: A Call for Renewed Commitment. Social Work with Groups, 39, 48-61. Recuperado de: https://www.tandfonline.com/doi/abs/10.1080/01609513.2014.989627Cembranos, F., \& Medina, J. Á. (2003). Grupos inteligentes. Teoría y práctica del trabajo en equipo. Madrid, España: Popular.

De Robertis, C. \& Pascal, H. (1994). La intervención colectiva en Trabajo Social. Buenos Aires, Argentina: El Ateneo.

Duro, J. C. (2002). El discurso de los profesionales de la atención primaria de la Comunidad de Madrid sobre el trabajo con grupos: sentido, finalidades y ámbitos de intervención. Revista Española de Salud Pública, vol. 76, nº 5, 545-559.

Drumm, K. (2006). The Essential Power of Group Work. Social Work with Groups, vol. 29, 17-31. Recuperado de: https://www.tandfonline.com/doi/abs/10.1300/J009v29n02_02

Fabris, F. A. (2007). Pichón-Rivière. Un viajero de mil mundos. Buenos Aires, Argentina: Polemos.

Flecha, R. (1997). Compartiendo palabras. El aprendizaje de las personas adultas a través del diálogo. Barcelona, España: Paidós.

Freire, P. (2007, [1970]). Pedagogía del oprimido. Madrid, España: Siglo XXI.

García Badaracco, J. E. (2000). Psicoanálisis multifamiliar. Los otros en nosotros y el descubrimiento del si mismo. Barcelona, España: Paidós.

Garrett, K. J. (2005). Use of Group in School Social Work: Group Work and Group Prorcesses. Social Work with Groups, 27:2-3, 75-92. Recuperado de: https://www.tandfonline.com/doi/abs/10.1300/ J009v27n02_06Grinberg, L., Sor, D. \& Tabak, E. (1991). Nueva introducción a la ideas de Bion. Madrid, España: Tecnipublicaciones.

Habermas, J. (2010). Teoría de la acción comunicativa. Madrid, España: Trotta.

Herranz, T., Silva, L. \& Herranz, M. (2014). Psicoterapia con niños y psicodrama: la cura por la alegría. Madrid, España: Síntesis.

Idareta, F. (2017). Eufemización de la violencia ejercida en trabajo social: Hacia el reconocimiento intradisciplinar del abuso de poder. Trabajo Social Hoy, núm. 81, 59-82.

Jiménez Bertomeu, V. (2002). Violencia en organizaciones y profesionales: acciones, omisiones y reacciones en torno al poder, la participación, la burocracia y la discreción de los Servicios Sociales. Alternativas. Cuadernos de Trabajo Social. No 10, 427-444.

Konopka, G. (1968). Trabajo social de grupo. Madrid, España: Euramérica.

La Roque, S. E. (2016). Group Work Education in Social Work: A Review of the Literature Reveals Possible Solutions. Journal of Social Work Education, Published online: 5 dec 2016, 1-10. Recuperado de: https://www.tandfonline.com/doi/abs/10.1080/10437797.2016.1246270 López-Barberá, E. \& Población, P. (s.f.) Apuntes de Psicodrama Moreniano. Recuperado de: http://grupopsicodrama.com/documents/Introducci\%C3\%B3nPsicodramaMoreno.pdf

López-Yarto, L. (2004, $1^{\text {a }}$ ed. 1997). Dinámica de grupos. Cincuenta años después. Bilbao, España: Desclée De Brouwer.

Moreno, J.L. (1993 [1961]). Psicodrama. Buenos Aires, Argentina: Lumen.

Papell, C. (2015). Social Work with Groups: What, Why, an Wherefore? Social Work with Groups,38, 241-246. Recuperado de: https://www.tandfonline.com/doi/abs/10.1080/01609513.2014.951163Parra Ramajo, B. (2014). Una revisión del trabajo social grupal. Revista de Treball Social, núm. 201, 23-29.

Pelegrí, X. (2004). El poder en el trabajo social: una aproximación desde Foucault. Cuadernos de Trabajo Social, núm. 17, 21-43.

Pereña, F. (2010). Soledad, pertenencia y transferencia. Madrid, España: Síntesis.

Pichón-Rivière, E. (1981). El proceso grupal. Del psicoanálisis a la psicología social (I). Buenos Aires, Argentina: Nueva Visión.

Rossell, T. (1998). Trabajo social de grupos socioterapéuticos y grupos socioeducativos. Cuadernos de Trabajo Social, núm. 11, 103-122.

Rossell, T. (2014). Método y modalidades del trabajo social de grupo. Revista de Treball Social, núm. 201 (abril), 9-22.

Roy, V. \& Pullen-Sanfaçon, A. (2016). Promoting Individual and Social Changes: A Hybrid Model of Social Work with Groups. Social Work whith Groups, 39, 4-40. Recuperado de: https://www.tandfonline. com/doi/abs/10.1080/01609513.2015.1033585Sánchez-Escárcega, J. (2006). El psicoanálisis grupal no es psicoanálisis individual. Revista Subjetividad y Cultura, de 16 de abril. Recuperado de: http://sub- 
jetividadycultura.org.mx/2013/04/el-psicoanalisis-grupal-: no-es-un-psicoanalisis-individual-en-publico-el-problema-de-la-interpretacion-en-el-grupo/

Shaw, M. E. (1976). Group Dynamics. New York, EEUU: McGraw-Hill.

Tannenbaum, N. \& Reisch, M. (2001). From Charitable Volunteers to Architects of Social Welfare: A Brief History of Social Work. Recuperado de: http://ssw.umich.edu/about/history/brief-history-of-social-work Todorov, T. (1995). La vida en común. Madrid, España: Taurus.

Vinogradov, S. \& Yalom, I. D. (2012). Guía breve de psicoterapia de grupo. Barcelona, España: Paidós.

Vives Belmonte, A. (2014). El grupo como herramienta de trabajo desde los servicios sociales. Revista de Trevall Social, núm. 201, 40-49.

Walling, D. J. (2012). El apego en psicoterapia. Bilbao, España: Desclée de Brower.

Zamanillo, T. \& Rodríguez, A. (1990). Un universo complejo. Los paradigmas en la intervención social. En Revista Documentación Social, Vol. 81, 9-33.

Zamanillo, T. (2008). Trabajo social con grupos y pedagogía ciudadana. Madrid, España: Síntesis.

Zamanillo, T. (2012). Las relaciones de poder en las profesiones de ayuda. Una cuestión ética de primer orden. Revista Internacional de Trabajo Social y Bienestar, Núm. 1, 157-170.

Zamanillo, T. (2014). Del grupo a la comunidad. El poder de la palabra. Revista de Treball Social, núm. 201, 50-65. 